\title{
Institutional Advances for Agriculture and Allied Activities in Karnataka with Special Emphasis on Investment Credit
}

\author{
V. K. Patil" and S. B. Mahajanashetti \\ Department of Agribusiness Management, College of Agriculture, UAS, Dharwad, \\ Karnataka-580005, India \\ *Corresponding author
}

\section{A B S T R A C T}

\begin{tabular}{l} 
K e y w o r d s \\
Agricultural credit, \\
Institutional \\
advances, \\
Investment loan, \\
Short term loan, \\
Term loan \\
Article Info \\
$\begin{array}{l}\text { Accepted: } \\
\text { 22 June } 2020 \\
\text { Available Online: } \\
\text { 10 July } 2020\end{array}$ \\
\hline
\end{tabular}

\section{Introduction}

Indian agriculture is gifted with fertile land and abundant availability of water resources. The prosperity of India depends mainly on the development of agriculture because a majority of population is engaged directly or indirectly in agriculture. Hence, the development of agriculture would mean the development of the rural masses and development of India. Further, the increase in agricultural productivity depends on the adoption of new farm practices such as high yielding variety seeds, chemical fertilizers, pesticides, farm machinery and assured irrigation facilities. The adoption of these practices depends on the availability of adequate amounts of finance. The creation of adequate and requisite credit avenues is therefore essential for agricultural progress. Credit plays the role of an accelerator for agricultural development. Capital investment for improvement in agricultural output over time makes the difference in performance of economies across countries. The importance of agricultural credit in a country like India which is essentially rural in nature hardly needs elaboration as the economic 
development of the countryside hinges upon the availability of credit to rural economy. Sustained agricultural growth has been a central theme of our developmental planning since independence for ensuring availability of and access to food. This however requires a consistent enhancement in public and private investment for sustained growth of agricultural sector of the country. The paper examines the changing share of term credit (investment credit) advanced to agriculture by different institutional credit agencies, that is, Scheduled Commercial Banks (SCBs), Cooperatives, and Regional Rural banks (RRBs) in North Karnataka specifically. The objectives of the present study are to analyze the flow of agriculture credit in India and Karnataka and study the current status of investment credit in North Karnataka.

\section{Materials and Methods}

Secondary data with respect to disbursement of agricultural credit in general and investment credit in particular from various financial institutions were collected from various sources like reports of RBI, district lead banks, NABARD, books and journals for the period 2005-06 to 2017-18.

\section{Analytical framework}

The collected data were analyzed to compute averages, percentages and compound annual growth rates.

\section{Growth rate analyses}

In order to analyze growth in total loan advances in study area, the following model was estimated:

$Y_{t}=A^{t} e^{U t} \ldots \ldots$

Where,
$\mathrm{Y}_{\mathrm{t}}=$ Credit disbursed during time $\mathrm{t}$

$\mathrm{A}=\mathrm{Y}$ in the base year

$\mathrm{t}=$ Time period

$\mathrm{U}_{\mathrm{t}}=$ Error term

$\mathrm{B}=1+\mathrm{g}$ where $\mathrm{g}=$ growth rate

By taking the logarithm, equation (1) was reduced to the following form,

$$
\ln Y_{t}=\ln A+(\ln B) t+U_{t}
$$

Where $\ln$ A and $\ln$ B were obtained by Ordinary Least Square (OLS) method

Once the above equation is estimated, $\mathrm{g}$ can be computed as:

$$
\mathrm{g}=[\operatorname{Antilog}(\mathrm{B})-1] * 100
$$

The estimated growth rates was tested for its significance using' $t$ ' statistic.

\section{Results and Discussion}

It can be seen from the table 1 that total agricultural advances including crop loan and term loan increased from₹ $1,80,104$ crores during 2005-06 to ₹ $11,62,617$ crores during 2017-18, with a growth rate of 17.03 per cent during 13 year period. Also, the advances of crop loan increased from ₹1, 05,282 crores during 2005-06 to ₹7, 53,214 crores during 2017-18, with a growth rate of 18.05 per cent. Similarly, term loan advances increased from $₹ 74,822$ crores to $₹ 4,09,403$ crores during the same period, with a growth rate of 14.89 per cent. It can also be inferred from the table that during the study period, the share of crop loan in the total advances was much higher than that of the term loan.

It can be also seen from the table that the total agricultural advances in Karnataka grew at 15.54 per cent during the above period, while crop loan and term loan grew at 16.65 per 
cent and 12.46 per cent respectively. In case of North Karnataka, the total agricultural advances, crop loans and term loans grew at 19.7 per cent, 20.00 per cent and 19.58 per cent respectively.

It is clear from the table that the growth in investment loan for agriculture was less than that in crop loan advances at all India level, Karnataka state and North Karnataka.

Table 2 depicts the percentage share of crop loan and term loan in total agricultural advances at all India level, Karnataka as well as in North Karnataka for each year during a period of 13 years from 2005-06 to 2017-18. The last row of the table presents average annual share of crop loan and term loan during the above period. It is clear from the table that average annual share of crop loan and term loan during the above period were 70.50 per cent and 29.50 per cent at all India level; 73.82 per cent and 26.18 per cent for Karnataka, and 75.76 per cent and 24.24 per cent for North Karnataka. This clearly indicated that the average share of investment credit in the total farm credit was less than 30 per cent at all India level, which was reflected in the farm credit portfolio for Karnataka as a whole and North Karnataka.

Table.1 Institutional Credit flow for agriculture and allied activities) at all India level, Karnataka and North Karnataka (Rs. in Crores)

\begin{tabular}{|c|c|c|c|c|c|c|c|c|c|}
\hline \multirow[t]{2}{*}{ Year } & \multicolumn{3}{|c|}{ All India } & \multicolumn{3}{|c|}{ Karnataka } & \multicolumn{3}{|c|}{ North Karnataka } \\
\hline & $\begin{array}{l}\text { Crop } \\
\text { loan }\end{array}$ & $\begin{array}{l}\text { Term } \\
\text { Loan }\end{array}$ & Total & $\begin{array}{c}\text { Crop } \\
\text { loan }\end{array}$ & $\begin{array}{l}\text { Term } \\
\text { Loan }\end{array}$ & Total & $\begin{array}{c}\text { Crop } \\
\text { loan }\end{array}$ & $\begin{array}{l}\text { Term } \\
\text { Loan }\end{array}$ & Total \\
\hline $2005-06$ & 105282 & 74822 & 180104 & 7943 & 4069 & 12013 & 3222.10 & 945.14 & 4167.24 \\
\hline $2006-07$ & 138455 & 90945 & 229400 & 9843 & 4457 & 14300 & 3576.00 & 1251.58 & 4827.55 \\
\hline $2007-08$ & 181393 & 73265 & 254658 & 10521 & 3920 & 14441 & 4379.43 & 1377.69 & 5757.12 \\
\hline 2008-09 & 210395 & 91287 & 301682 & 12677 & 4608 & 17285 & 4017.52 & 1449.06 & 5466.59 \\
\hline $2009-10$ & 276656 & 107858 & 384514 & 15822 & 5521 & 21343 & 5224.06 & 1704.97 & 6929.03 \\
\hline 2010-11 & 319108 & 127671 & 446779 & 17982 & 8168 & 26150 & 5661.33 & 1983.19 & 7644.51 \\
\hline 2011-12 & 396158 & 114871 & 511029 & 18144 & 6221 & 24365 & 8293.76 & 2742.27 & 11036.03 \\
\hline $2012-13$ & 473500 & 133875 & 607375 & 26984 & 8602 & 35586 & 14380.61 & 4462.97 & 18843.58 \\
\hline 2013-14 & 573001 & 138620 & 711621 & 28728 & 7739 & 36467 & 13232.07 & 3551.74 & 16783.81 \\
\hline 2014-15 & 635412 & 209916 & 845328 & 34529 & 7913 & 42441 & 16512.05 & 3956.77 & 20468.82 \\
\hline $2015-16$ & 665313 & 250197 & 915510 & 39358 & 12187 & 51545 & 18113.14 & 6385.15 & 24498.30 \\
\hline 2016-17 & 689457 & 376298 & 1065756 & 44349 & 12257 & 56606 & 22956.32 & 5123.34 & 28079.67 \\
\hline 2017-18 & 753214 & 409403 & 1162617 & 46655 & 19575 & 66230 & 20765.86 & 9857.24 & 30623.10 \\
\hline $\begin{array}{c}\text { Average } \\
\text { amount } \\
\text { per annum }\end{array}$ & 416719 & 169156 & 535875 & 24118 & 8095 & 32213 & 10794.94 & 3445.47 & 14240.41 \\
\hline $\begin{array}{l}\text { Compound } \\
\text { annual } \\
\text { growth } \\
\text { rate } \\
\text { (CAGR) }\end{array}$ & $18.05 * *$ & $14.89 * *$ & $17.03 * *$ & $16.65 * *$ & $12.46 * *$ & $15.54 * *$ & $20.00 * *$ & $19.58 * *$ & $19.70 * *$ \\
\hline
\end{tabular}

Source: NABARD reports

Note: - ** Significant at $1 \%$ level 
Table.2 Percentage share of crop loan and term loan in total advances for agriculture and allied activities all India level, Karnataka and North Karnataka (Per cent)

\begin{tabular}{|c|c|c|c|c|c|c|}
\hline \multirow{2}{*}{ Years } & \multicolumn{2}{|c|}{ India } & \multicolumn{2}{c|}{ Karnataka } & \multicolumn{2}{c|}{ North Karnataka } \\
\cline { 2 - 7 } & $\begin{array}{c}\text { Crop } \\
\text { loan }\end{array}$ & $\begin{array}{c}\text { Term } \\
\text { loan }\end{array}$ & $\begin{array}{c}\text { Crop } \\
\text { loan }\end{array}$ & $\begin{array}{c}\text { Term } \\
\text { loan }\end{array}$ & $\begin{array}{c}\text { Crop } \\
\text { loan }\end{array}$ & $\begin{array}{c}\text { Term } \\
\text { loan }\end{array}$ \\
\hline $2005-06$ & 58.46 & 41.54 & 66.12 & 33.88 & 77.32 & 22.68 \\
\hline $2006-07$ & 60.36 & 39.64 & 68.83 & 31.17 & 74.07 & 25.93 \\
\hline $2007-08$ & 71.23 & 28.77 & 72.85 & 27.15 & 76.07 & 23.93 \\
\hline $2008-09$ & 69.74 & 30.26 & 73.34 & 26.66 & 73.49 & 26.51 \\
\hline $2009-10$ & 71.95 & 28.05 & 74.13 & 25.87 & 75.39 & 24.61 \\
\hline $2010-11$ & 71.42 & 28.58 & 68.76 & 31.24 & 74.06 & 25.94 \\
\hline $2011-12$ & 77.52 & 22.48 & 74.47 & 25.53 & 75.15 & 24.85 \\
\hline $2012-13$ & 77.96 & 22.04 & 75.83 & 24.17 & 76.32 & 23.68 \\
\hline $2013-14$ & 80.52 & 19.48 & 78.78 & 21.22 & 78.84 & 21.16 \\
\hline $2014-15$ & 75.17 & 24.83 & 81.36 & 18.64 & 80.67 & 19.33 \\
\hline $2015-16$ & 72.67 & 27.33 & 76.36 & 23.64 & 73.94 & 26.06 \\
\hline $2016-17$ & 64.69 & 35.21 & 78.35 & 21.65 & 81.75 & 18.25 \\
\hline $2017-18$ & 64.79 & 29.50 & 70.44 & 29.56 & 67.81 & 32.19 \\
\hline Average over years & 70.50 & 29.50 & 73.82 & 26.18 & 75.76 & 24.24 \\
\hline
\end{tabular}

Table.3 (A) Agency wise share of crop loan and term loan in total institutional agricultural advances at all India level (Per cent)

\begin{tabular}{|c|c|c|c|c|c|c|c|c|}
\hline \multirow{2}{*}{ Years } & \multicolumn{2}{|c|}{ Commercial bank } & \multicolumn{2}{|c|}{ Cooperative bank } & \multicolumn{2}{|c|}{ RRBs } & \multicolumn{2}{c|}{ Total } \\
\hline & $\begin{array}{c}\text { Crop } \\
\text { loan }\end{array}$ & $\begin{array}{c}\text { Term } \\
\text { loan }\end{array}$ & $\begin{array}{c}\text { Crop } \\
\text { term }\end{array}$ & $\begin{array}{c}\text { Term } \\
\text { loan }\end{array}$ & $\begin{array}{c}\text { Crop } \\
\text { term }\end{array}$ & $\begin{array}{c}\text { Term } \\
\text { loan }\end{array}$ & $\begin{array}{c}\text { Crop } \\
\text { term }\end{array}$ & $\begin{array}{c}\text { Term } \\
\text { loan }\end{array}$ \\
\hline $\mathbf{2 0 0 5 - 0 6}$ & 54.75 & 90.66 & 33.18 & 5.98 & 12.07 & 3.36 & 100 & 100 \\
\hline $\mathbf{2 0 0 6 - 0 7}$ & 60.09 & 91.58 & 27.89 & 4.24 & 12.01 & 4.18 & 100 & 100 \\
\hline $\mathbf{2 0 0 7 - 0 8}$ & 64.48 & 87.52 & 23.87 & 6.78 & 11.65 & 5.70 & 100 & 100 \\
\hline $\mathbf{2 0 0 8 - 0 9}$ & 70.26 & 88.88 & 19.09 & 6.36 & 10.65 & 4.77 & 100 & 100 \\
\hline $\mathbf{2 0 0 9 - 1 0}$ & 68.64 & 88.91 & 20.58 & 6.07 & 10.77 & 5.02 & 100 & 100 \\
\hline $\mathbf{2 0 1 0 - 1 1}$ & 67.93 & 90.81 & 20.22 & 4.37 & 11.85 & 4.82 & 100 & 100 \\
\hline $\mathbf{2 0 1 1 - 1 2}$ & 67.38 & 88.52 & 20.66 & 5.34 & 11.97 & 6.14 & 100 & 100 \\
\hline $\mathbf{2 0 1 2 - 1 3}$ & 66.52 & 87.80 & 21.67 & 6.43 & 11.82 & 5.77 & 100 & 100 \\
\hline $\mathbf{2 0 1 3 - 1 4}$ & 67.84 & 86.77 & 19.82 & 4.61 & 12.34 & 8.63 & 100 & 100 \\
\hline $\mathbf{2 0 1 4 - 1 5}$ & 65.43 & 89.86 & 20.51 & 3.87 & 14.06 & 6.27 & 100 & 100 \\
\hline $\mathbf{2 0 1 5 - 1 6}$ & 63.12 & 89.14 & 21.61 & 3.79 & 15.27 & 7.07 & 100 & 100 \\
\hline $\mathbf{2 0 1 6 - 1 7}$ & 65.64 & 92.27 & 19.13 & 2.89 & 15.23 & 4.84 & 100 & 100 \\
\hline $\mathbf{2 0 1 7}-18$ & 66.03 & 91.29 & 18.07 & 3.47 & 15.90 & 5.23 & 100 & 100 \\
\hline Average & $\mathbf{6 5 . 2 4}$ & $\mathbf{8 9 . 5 4}$ & $\mathbf{2 2 . 0 2}$ & $\mathbf{4 . 9 4}$ & $\mathbf{1 2 . 7 4}$ & $\mathbf{5 . 5 2}$ & $\mathbf{1 0 0}$ & $\mathbf{1 0 0}$ \\
\hline & & & & & & & \\
\hline
\end{tabular}


Table.3 (B) Agency wise share of crop loan and term loan in total institutional agricultural advances in Karnataka (Per cent)

\begin{tabular}{|c|c|c|c|c|c|c|c|c|}
\hline \multirow{2}{*}{ Years } & \multicolumn{2}{|c|}{ Commercial bank } & \multicolumn{2}{|c|}{ Cooperative bank } & \multicolumn{2}{|c|}{ RRBs } & \multicolumn{2}{c|}{ Total } \\
\cline { 2 - 10 } & $\begin{array}{c}\text { Crop } \\
\text { loan }\end{array}$ & $\begin{array}{c}\text { Term } \\
\text { loan }\end{array}$ & $\begin{array}{c}\text { Crop } \\
\text { term }\end{array}$ & $\begin{array}{c}\text { Term } \\
\text { loan }\end{array}$ & $\begin{array}{c}\text { Crop } \\
\text { term }\end{array}$ & $\begin{array}{c}\text { Term } \\
\text { loan }\end{array}$ & $\begin{array}{c}\text { Crop } \\
\text { term }\end{array}$ & $\begin{array}{c}\text { Term } \\
\text { loan }\end{array}$ \\
\hline $\mathbf{2 0 0 5 - 0 6}$ & 52.93 & 87.23 & 29.12 & 5.60 & 17.95 & 7.16 & 100 & 100 \\
\hline $\mathbf{2 0 0 6 - 0 7}$ & 59.08 & 83.77 & 21.16 & 8.14 & 19.76 & 8.09 & 100 & 100 \\
\hline $\mathbf{2 0 0 7 - 0 8}$ & 50.94 & 83.32 & 26.52 & 7.30 & 22.54 & 9.38 & 100 & 100 \\
\hline $\mathbf{2 0 0 8 - 0 9}$ & 53.05 & 85.90 & 23.75 & 6.21 & 23.20 & 7.88 & 100 & 100 \\
\hline $\mathbf{2 0 0 9 - 1 0}$ & 56.84 & 85.59 & 22.62 & 6.29 & 20.54 & 8.12 & 100 & 100 \\
\hline $\mathbf{2 0 1 0 - 1 1}$ & 58.58 & 88.20 & 23.64 & 5.04 & 17.78 & 6.76 & 100 & 100 \\
\hline $\mathbf{2 0 1 1 - 1 2}$ & 52.47 & 71.08 & 28.65 & 13.13 & 18.89 & 15.79 & 100 & 100 \\
\hline $\mathbf{2 0 1 2}-13$ & 52.68 & 69.66 & 33.84 & 12.40 & 13.48 & 17.94 & 100 & 100 \\
\hline $\mathbf{2 0 1 3 - 1 4}$ & 58.28 & 78.10 & 25.29 & 8.18 & 16.43 & 13.71 & 100 & 100 \\
\hline $\mathbf{2 0 1 4 - 1 5}$ & 56.63 & 67.70 & 24.75 & 15.32 & 18.62 & 16.98 & 100 & 100 \\
\hline $\mathbf{2 0 1 5 - 1 6}$ & 59.26 & 75.00 & 23.13 & 8.65 & 17.61 & 16.35 & 100 & 100 \\
\hline $\mathbf{2 0 1 6 - 1 7}$ & 61.68 & 76.07 & 20.15 & 8.21 & 18.17 & 15.72 & 100 & 100 \\
\hline $\mathbf{2 0 1 7}-18$ & 61.56 & 72.29 & 22.71 & 8.49 & 15.74 & 19.22 & 100 & 100 \\
\hline Average & $\mathbf{5 6 . 4 6}$ & $\mathbf{7 8 . 7 6}$ & $\mathbf{2 5 . 0 2}$ & $\mathbf{8 . 6 9}$ & $\mathbf{1 8 . 5 2}$ & $\mathbf{1 2 . 5 5}$ & $\mathbf{1 0 0}$ & $\mathbf{1 0 0}$ \\
\hline over years & & & & & & & & \\
\hline
\end{tabular}

Table.3 (C) Agency wise share of crop loan and term loan in total institutional agricultural advances in North Karnataka (Per cent)

\begin{tabular}{|c|c|c|c|c|c|c|c|c|}
\hline \multirow{2}{*}{ Years } & \multicolumn{2}{|c|}{ Commercial bank } & \multicolumn{2}{c|}{ Cooperative bank } & \multicolumn{2}{c|}{ RRBs } & \multicolumn{2}{c|}{ Total } \\
\cline { 2 - 9 } & $\begin{array}{c}\text { Crop } \\
\text { loan }\end{array}$ & $\begin{array}{c}\text { Term } \\
\text { loan }\end{array}$ & $\begin{array}{c}\text { Crop } \\
\text { term }\end{array}$ & $\begin{array}{c}\text { Term } \\
\text { loan }\end{array}$ & $\begin{array}{c}\text { Crop } \\
\text { term }\end{array}$ & $\begin{array}{c}\text { Term } \\
\text { loan }\end{array}$ & $\begin{array}{c}\text { Crop } \\
\text { term }\end{array}$ & $\begin{array}{c}\text { Term } \\
\text { loan }\end{array}$ \\
\hline $\mathbf{2 0 0 5 - 0 6}$ & 35.58 & 74.43 & 40.19 & 8.19 & 24.22 & 17.38 & 100 & 100 \\
\hline $\mathbf{2 0 0 6 - 0 7}$ & 39.86 & 71.24 & 31.19 & 9.85 & 28.94 & 18.90 & 100 & 100 \\
\hline $\mathbf{2 0 0 7 - 0 8}$ & 38.51 & 72.58 & 34.16 & 9.09 & 27.32 & 18.33 & 100 & 100 \\
\hline $\mathbf{2 0 0 8 - 0 9}$ & 39.22 & 61.42 & 34.37 & 15.32 & 26.41 & 23.26 & 100 & 100 \\
\hline $\mathbf{2 0 0 9}-10$ & 41.69 & 59.58 & 28.05 & 8.57 & 30.26 & 31.86 & 100 & 100 \\
\hline $\mathbf{2 0 1 0}-11$ & 35.64 & 57.19 & 30.14 & 15.00 & 34.22 & 27.81 & 100 & 100 \\
\hline $\mathbf{2 0 1 1 - 1 2}$ & 44.50 & 59.74 & 33.05 & 10.78 & 22.44 & 29.48 & 100 & 100 \\
\hline $\mathbf{2 0 1 2}-13$ & 40.10 & 58.09 & 47.08 & 12.86 & 12.82 & 29.06 & 100 & 100 \\
\hline $\mathbf{2 0 1 3 - 1 4}$ & 51.70 & 68.22 & 29.48 & 9.73 & 18.82 & 22.05 & 100 & 100 \\
\hline $\mathbf{2 0 1 4 - 1 5}$ & 48.94 & 64.12 & 30.08 & 14.13 & 20.98 & 21.76 & 100 & 100 \\
\hline $\mathbf{2 0 1 5}-16$ & 55.57 & 75.32 & 26.08 & 8.13 & 18.35 & 16.56 & 100 & 100 \\
\hline $\mathbf{2 0 1 6}-17$ & 59.72 & 71.02 & 21.77 & 9.07 & 18.51 & 19.91 & 100 & 100 \\
\hline $\mathbf{2 0 1 7}-18$ & 61.71 & 62.41 & 22.01 & 10.23 & 16.29 & 27.36 & 100 & 100 \\
\hline Average & $\mathbf{4 5 . 6 0}$ & $\mathbf{6 5 . 8 0}$ & $\mathbf{3 1 . 3 6}$ & $\mathbf{1 0 . 8 4}$ & $\mathbf{2 3 . 0 4}$ & $\mathbf{2 3 . 3 6}$ & $\mathbf{1 0 0}$ & $\mathbf{1 0 0}$ \\
\hline over years & & & & & & & & \\
\hline
\end{tabular}


Table.4 (A) Share of farm investment activities in total term loan for agriculture in Karnataka (Per cent)

\begin{tabular}{|c|c|c|c|c|c|c|c|c|c|c|c|c|c|c|}
\hline Year & $\begin{array}{c}\text { Minor } \\
\text { Irrigation }\end{array}$ & $\begin{array}{c}\text { Land } \\
\text { Development }\end{array}$ & $\begin{array}{c}\text { Farm } \\
\text { Mechanization }\end{array}$ & $\begin{array}{l}\text { Plantation \& } \\
\text { Horticulture }\end{array}$ & $\begin{array}{c}\text { Dairy } \\
\text { Development }\end{array}$ & Poultry & $\begin{array}{c}\text { Sheep } \\
\text { Goat and } \\
\text { Piggery }\end{array}$ & Fisheries & $\begin{array}{c}\text { Forestry and } \\
\text { Waste land } \\
\text { management }\end{array}$ & $\begin{array}{c}\text { Storage } \\
\text { godown and } \\
\text { market yard }\end{array}$ & $\begin{array}{l}\text { Bio } \\
\text { gas }\end{array}$ & Sericulture & Others & Total \\
\hline 2011-12 & 13.51 & 10.45 & 9.81 & 19.17 & 8.34 & 1.29 & 1.18 & 0.50 & 1.20 & 7.92 & 0.00 & 0.01 & 26.62 & 100.00 \\
\hline 2012-13 & 12.70 & 8.07 & 9.96 & 19.92 & 6.20 & 2.45 & 1.52 & 1.86 & 2.46 & 5.51 & 0.00 & 0.00 & 29.35 & 100.00 \\
\hline 2013-14 & 15.18 & 10.04 & 12.36 & 14.33 & 7.52 & 2.13 & 1.74 & 11.74 & 2.24 & 5.10 & 0.00 & 0.02 & 17.60 & 100.00 \\
\hline 2014-15 & 14.30 & 10.20 & 11.65 & 18.90 & 7.01 & 2.46 & 1.54 & 2.22 & 0.98 & 3.87 & 0.00 & 0.04 & 26.83 & 100.00 \\
\hline 2015-16 & 11.79 & 11.61 & 10.74 & 13.99 & 10.36 & 2.97 & 2.05 & 3.59 & 0.66 & 4.94 & 0.03 & 0.00 & 27.26 & 100.00 \\
\hline 2016-17 & 8.30 & 9.83 & 8.82 & 11.93 & 8.92 & 2.78 & 2.84 & 1.94 & 1.41 & 4.84 & 0.01 & 0.00 & 38.37 & 100.00 \\
\hline 2017-18 & 12.02 & 10.75 & 9.21 & 12.77 & 11.21 & 2.75 & 3.78 & 3.44 & 0.95 & 3.01 & 0.02 & 0.00 & 30.08 & 100.00 \\
\hline $\begin{array}{c}\text { Average } \\
\text { over } \\
\text { years }\end{array}$ & 12.54 & 10.14 & 10.37 & 15.86 & 8.51 & 2.41 & 2.09 & 3.61 & 1.41 & 5.03 & 0.01 & 0.01 & 28.01 & 100.00 \\
\hline
\end{tabular}

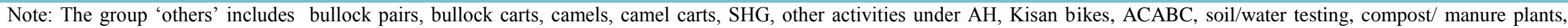

vermiculture, bee- keeping, etc.

Table.4 (B) Share of farm investment activities in total term loan for Agriculture in North Karnataka (Per cent)

\begin{tabular}{|c|c|c|c|c|c|c|c|c|c|c|c|c|c|c|}
\hline Year & $\begin{array}{c}\text { Minor } \\
\text { Irrigation }\end{array}$ & $\begin{array}{c}\text { Land } \\
\text { Development }\end{array}$ & $\begin{array}{c}\text { Farm } \\
\text { Mechanization }\end{array}$ & $\begin{array}{c}\text { Plantation } \\
\& \\
\text { Horticulture }\end{array}$ & $\begin{array}{c}\text { Dairy } \\
\text { Development }\end{array}$ & Poultry & $\begin{array}{c}\text { Sheep } \\
\text { Goat and } \\
\text { Piggery }\end{array}$ & Fisheries & $\begin{array}{c}\text { Forestry } \\
\text { and Waste } \\
\text { land } \\
\text { management }\end{array}$ & $\begin{array}{c}\text { Storage } \\
\text { godown and } \\
\text { market } \\
\text { yard }\end{array}$ & $\begin{array}{l}\text { Bio } \\
\text { gas }\end{array}$ & Sericulture & Others & Total \\
\hline 2005-06 & 9.85 & 8.60 & 40.57 & 9.85 & 5.51 & 0.75 & 0.92 & 0.33 & 0.74 & 9.40 & 0.05 & 0.17 & 13.28 & 100.00 \\
\hline 2006-07 & 10.05 & 9.37 & 30.14 & 8.86 & 3.74 & 1.32 & 0.80 & 0.29 & 0.59 & 14.82 & 0.03 & 0.16 & 19.83 & 100.00 \\
\hline 2007-08 & 9.21 & 8.84 & 24.86 & 9.04 & 5.76 & 0.68 & 0.92 & 0.36 & 0.75 & 10.69 & 0.00 & 0.18 & 28.70 & 100.00 \\
\hline 2008-09 & 11.18 & 10.31 & 17.20 & 7.55 & 4.92 & 1.08 & 0.90 & 2.05 & 4.96 & 14.34 & 0.01 & 0.16 & 25.35 & 100.00 \\
\hline 2009-10 & 14.22 & 7.92 & 20.79 & 7.48 & 3.16 & 0.90 & 0.54 & 0.52 & 0.64 & 20.66 & 0.00 & 0.10 & 23.06 & 100.00 \\
\hline 2010-11 & 22.20 & 7.83 & 15.46 & 6.13 & 7.49 & 2.59 & 0.85 & 0.94 & 2.77 & 5.10 & 0.00 & 0.02 & 28.62 & 100.00 \\
\hline 2011-12 & 25.59 & 6.07 & 13.84 & 4.20 & 10.75 & 0.73 & 1.21 & 0.51 & 2.10 & 11.17 & 0.00 & 0.01 & 23.82 & 100.00 \\
\hline 2012-13 & 20.55 & 7.79 & 13.23 & 5.49 & 4.80 & 0.94 & 0.75 & 0.46 & 3.07 & 6.83 & 0.00 & 0.00 & 36.10 & 100.00 \\
\hline 2013-14 & 28.62 & 13.16 & 18.97 & 6.97 & 5.20 & 0.82 & 1.68 & 0.27 & 1.47 & 9.36 & 0.00 & 0.00 & 13.48 & 100.00 \\
\hline 2014-15 & 17.85 & 6.56 & 16.92 & 9.85 & 5.29 & 1.02 & 1.54 & 0.57 & 1.66 & 6.67 & 0.00 & 0.00 & 32.07 & 100.00 \\
\hline 2015-16 & 18.93 & 11.61 & 14.45 & 9.08 & 7.07 & 2.78 & 1.86 & 0.14 & 0.75 & 7.85 & 0.06 & 0.00 & 25.40 & 100.00 \\
\hline 2016-17 & 14.02 & 13.38 & 13.62 & 11.77 & 9.25 & 4.17 & 2.96 & 2.20 & 2.59 & 7.26 & 0.03 & 0.00 & 18.74 & 100.00 \\
\hline 2017-18 & 20.16 & 10.77 & 14.11 & 8.90 & 12.23 & 1.59 & 5.03 & 0.74 & 1.61 & 3.60 & 0.05 & 0.00 & 21.21 & 100.00 \\
\hline $\begin{array}{c}\text { Average over } \\
\text { years }\end{array}$ & 17.11 & 9.40 & 19.55 & 8.09 & 6.55 & 1.49 & 1.54 & 0.72 & 1.82 & 9.83 & 0.02 & 0.06 & 23.82 & 100.00 \\
\hline
\end{tabular}


Table 3 (A) indicates the share of crop loans and term loans advanced by commercial banks, cooperative banks and RRBs in the total institutional advances of crop loan and term loan in India. Similarly, tables 3 (B) and 3 (C) give this picture in respect of Karnataka and North Karnataka respectively. In table 3 (A), the shares for the country as a whole are given for each of the 13 years between 2005 06 and 2017-18. The last row of the table presents the average annual shares during the above period. It can be made out from the table that in the total crop loan advances in the country, the share of commercial banks on an average was around $65.24 \%$, while cooperative banks and RRBs accounted for $22.02 \%$ and $12.74 \%$. Even in the case of term loan, the commercial banks had a major share of $89.54 \%$ with cooperatives and RRBs accounting for $4.94 \%$ and $5.52 \%$ respectively. The trend was similar in respect of Karnataka state also as can be seen from table 3 (B). In the state, commercial banks, cooperatives and RRBs had a share of 56.46 $\%, 25.02 \%$ and $18.52 \%$ in the total crop loan advances in the country. With respect to the total term loan advances in the country, the share of commercial banks, cooperatives and RRBs was $78.76 \%, 8.69 \%$ and $12.55 \%$ respectively. Similar trend could be seen in North Karnataka as well. From these results, it was clear that the commercial banks had maximum share followed by cooperatives and RRBs in respect of total crop loan advances in the country. In the case of term loans, it was again commercial banks, which had maximum share. However, in respect of term loans RRBs had the second highest share unlike in crop loan.

Tables 4 (A) presents the shares of individual investment activities in the total agriculture term loan in Karnataka for 7 years from 201112 to 2017-18. Table 4 (B) presents this information for North Karnataka.

From the results presented in the table 4 (A) pertaining to Karnataka, it is evident that the highest average share of term loan was that of plantation and horticulture $(15.86 \%)$ followed by minor irrigation (12.54\%), farm mechanization (10.37\%), land development (10.14\%), dairy development (8.51\%), storage go down and market yard (5.03\%), fishery $(3.61 \%)$, poultry $(2.41 \%)$, sheep goat and piggery $(2.09 \%)$ and forestry and waste land development (1.41\%).

From the results presented in the table 4 (B) pertaining to North Karnataka, it is evident that the highest average share of term loan was that of farm mechanization (19.55\%) followed by minor irrigation (17.11\%), storage go down and market yard $(9.83 \%)$, land development $(9.40 \%)$, plantation and horticulture (8.09\%), dairy development (6.55\%), forestry and waste land development (1.82\%), sheep goat and piggery (1.54\%), poultry $(1.49 \%)$, and fishery $(0.72 \%)$.

In conclusion during the study period, the growth in investment loan for agriculture was less than that of crop loan at all India level, Karnataka state level and for North Karnataka. The share of term loan in the total agricultural advances was less than 30 per cent at all the three levels considered. Commercial banks are playing a dominant role in advancing crop loan as well as term loan. In Karnataka, plantation and horticulture had maximum share in the term loan for agriculture. However, when examining the situation for North Karnataka it was clear that, farm mechanization accounted for maximum share in the total term loan for agriculture.

\section{References}

Awasathi, A, Production and Investment Credit of Scheduled Commercial Banks in India: Need for a Systematic Approach. Indian.Journal of Agricultural Economics. 2007; 
62(3):314-320.

Dar, J.A, Trend and Growth of Flow of Credit to Agriculture after 1991 in India. Journal of Humanities and Social Sciences Studies. 2015;20(1):51-61.

Godara, R. L, Singh, P. and Singla S, Agriculture Credit in India: An Analytical study. International Journal of Latest Trends in Engineering and Technology. 2014; 3(3):326-335.

Panda, R.K, Investment Behavior of Farm Household and Flow of Investment Credit in Orissa. Indian.Journal of Agricultural Economics. 2005;
60(3):399.

Prakash, Brahm, Srivastava, R. K, Sharma, D. K. and Khare A. P, Growth of Agricultural Credit in India: Trends and Problems. Indian. Journal of Agricultural Economics.2007; 62(3): 397.

Shukla, A.N, Tewari, S.K., and Dubey, P. P, An Analysis of Status and Trends of Investment Credit in Indian Agriculture. Journal of Agricultural Sciences.2012; 3(1): 29-33.

\section{How to cite this article:}

Patil, V. K. and Mahajanashetti, S. B. 2020. Institutional Advances for Agriculture and Allied Activities in Karnataka with Special Emphasis on Investment Credit. Int.J.Curr.Microbiol.App.Sci. 9(07): 3044-3051. doi: https://doi.org/10.20546/ijcmas.2020.907.359 